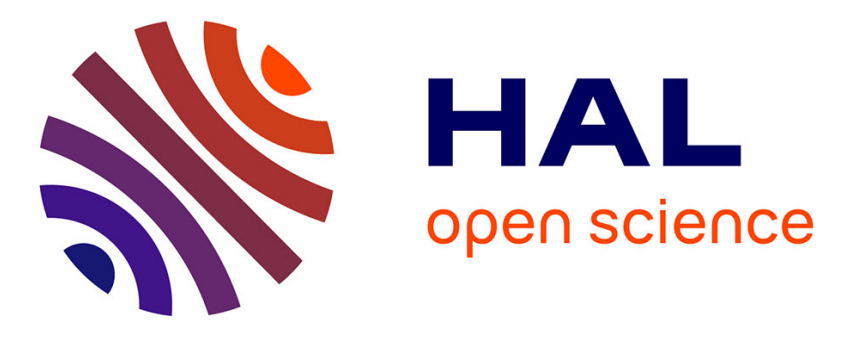

\title{
Formation and optical response of self-assembled gold nanoparticle lattices on oxidized silicon synthesized using block copolymers
}

\author{
Alberto Alvarez-Fernandez, Guillaume Fleury, Virginie Ponsinet, Per Magnus \\ Walmsness, Morten Kildemo
}

\section{To cite this version:}

Alberto Alvarez-Fernandez, Guillaume Fleury, Virginie Ponsinet, Per Magnus Walmsness, Morten Kildemo. Formation and optical response of self-assembled gold nanoparticle lattices on oxidized silicon synthesized using block copolymers. Journal of Vacuum Science \& Technology B, Nanotechnology and Microelectronics, 2020, 38 (1), pp.013601. 10.1116/1.5129667 . hal-02439559

\section{HAL Id: hal-02439559 \\ https://hal.science/hal-02439559}

Submitted on 10 Nov 2020

HAL is a multi-disciplinary open access archive for the deposit and dissemination of scientific research documents, whether they are published or not. The documents may come from teaching and research institutions in France or abroad, or from public or private research centers.
L'archive ouverte pluridisciplinaire HAL, est destinée au dépôt et à la diffusion de documents scientifiques de niveau recherche, publiés ou non, émanant des établissements d'enseignement et de recherche français ou étrangers, des laboratoires publics ou privés. 


\title{
Formation and optical response of self-assembled gold nanoparticle lattices on oxidized silicon synthesized using block copolymers
}

\author{
A. Alvarez-Fernandez \\ CNRS, Univ. Bordeaux, Centre de Recherche Paul Pascal, \\ UMR 5031, 115 Avenue Schweitzer, 33600 Pessac, France and \\ CNRS, Laboratoire de Chimie des Polymères Organiques, \\ UMR 5629 - ENSCBP - Univ. Bordeaux, 16 Avenue Pey-Berland, 33607 Pessac Cedex, France \\ G. Fleury \\ CNRS, Laboratoire de Chimie des Polymères Organiques, \\ UMR 5629 - ENSCBP - Univ. Bordeaux, 16 Avenue Pey-Berland, 33607 Pessac Cedex, France \\ V. Ponsinet \\ CNRS, Univ. Bordeaux, Centre de Recherche Paul Pascal, \\ UMR 5031, 115 Avenue Schweitzer, 33600 Pessac, France \\ P.M. Walmsness and M. Kildemo* \\ Department of Physics, NTNU Norwegian University of Science and Technology, NO-7491 Trondheim, Norway
}

(Dated: November 10, 2020)

\begin{abstract}
We report here on the optical response and extraction of the morphological properties of three sizes of self assembled nearly hexagonal arrays of gold $(\mathrm{Au})$ nanoparticles, deposited on native oxide on c-Si. The arrays were prepared by templating gold NP growth with self-assembled cylindrically organized block copolymers thin films, with consecutive removal of the polymer matrix by plasma etching. The particle sizes were controlled by using block copolymers of different molecular lengths when assembling the templates. The resulting gold nanostructures were characterized by scanning probe microscopy. Spectroscopic ellipsometry was used to record the optical response of the samples, and the modelling of the spectra and the extraction of morphological parameters were performed using a modification of the Bedeaux-Vlieger formalism implemented in GranFILM. The modelling issues upon introduction of a thin intermediate (oxyde) layer between the particle and the substrate is discussed in detail, and a solution to the model procedure is proposed. The particles were modelled as truncated oblate $\mathrm{Au}$ spheroids in a hexagonal lattice on native oxide on c-Si. The model fit converged to a solution indicating that the particles present a low wetting of the native oxide substrate, with parameters describing the particles and lattice in good correspondence with AFM. The extended GranFiLm model simulations are also supported by Finite Element Modelling.
\end{abstract}

\section{INTRODUCTION}

Nanotechnology was seeded by the idea of versatile material synthesis by direct manipulation and assembly of atoms or elementary entities [1]. It has unraveled nanosize-related enhanced or even unique mechanical, catalytic, electrical and optical properties; and has led to an exponential growth of new engineered nanomaterials and their exploitation by growing industries in microelectronics, medicine, energy, etc [2]. It has developed in the capacities to achieve a wide range of functions and structures, which are in the process of revolutionizing many aspects of our lives. In the field of optics and imaging, the pace of evolution is specifically high $[3,4]$, with nanophotonics exploring the possibility of modulating light propagation with very small amount of matter using nanoscale phenomena. Diffractive effects are involved when characteristic sizes are of the order of the wavelength of light, while optical resonances are used in

* morten.kildemo@ntnu.no metamaterials structured at subwavelength dimensions. Optical metamaterials are indeed artificial nanomaterials designed and engineered in order to propagate light in a non-natural manner. Their advent for the last twenty years relies on the recent capacities of both numerical simulations and nanofabrication, which makes it possible to produce nanostructures tailored to present defined resonances upon light illumination. Of special interest are regular arrays of individual metal nanoresonators, presenting upon light illumination, the resonance of collective oscillations of the conduction electrons, i.e. localized surface plasmon resonances (LSPRs). This phenomenon induces a subwavelength confinement of the optical energy, of critical importance for many applications such as sensitive detectors, optical filters, waveguides, and other photonic-circuit components [5-7]. Nevertheless, in spite of invaluable progress in nanofabrication techniques for nanoscale geometrical features of metallic and dielectric nature, we are still in need of fabrication routes allowing the production of large areas or volumes, with good degree of structure control, and low cost and low environmental impact. This is why so-called "bottom-up" methodologies, based on chemistry and self- 
assembly, have triggered significant interest in recent years [8-10]. Among the promising routes, is the use of the self-assembly of block copolymers (BCPs) into nanostructured materials, which has been described for many years [11-13]. Diblock copolymers are macromolecules made of two polymer chains of distinct chemical nature covalently linked, called the blocks, and present solid state spontaneous structuration with long-range order and tunable characteristic sizes, ranging typically from a few nanometers to a few hundred nanometers. They have been proposed in the recent years as templates for the fabrication of metamaterials, metasurfaces and other nanostructures with optical functions [14-20]. Their capacity to easily produce controlled nanoparticle arrays will be used in the work presented here. Metal nanoparticle arrays constitute a simple but promising platform for manipulating light-matter interactions [5]. The optical study of both top-down and bottom-up plasmonic nanostructured thin films has stimulated many theoretical and experimental studies ever since Maxwell-Garnett [21-23].

For truncated particles supported by a substrate, the asymmetry due to the presence of the substrate strongly complicates the modelling. The Bedeaux Vlieger (BV) formalism is a powerful method for dealing with such particles that are partially truncated [24]. In this paper, the most recent implementation (GRANFILM software) of the BV theory is used to model regular lattices of truncated metal ellipsoids (or more correctly spheroids, as they are in-plane isotropic) supported on a flat dielectric substrate [25]. However, such samples are commonly prepared on c-Si substrates covered with a thin native oxide layer, as all samples reported here. In this paper we thus propose a modification of the $\mathrm{BV}$ formalism in order to include the underlying thin film structure $\left(\mathrm{SiO}_{2}\right.$ layer on c-Si substrate).

\section{EXPERIMENTAL}

\section{A. Thin film preparation}

During this work, several poly(styrene)-b-poly(4vinylpyridine) (PS-b-P4VP) and poly(styrene)-b-poly (2vinylpyridine) (PS-b-P2VP) BCPs have been synthesized by living anionic polymerization, according to the standard procedure reported in the literature $[26,27]$ and presented in Fig. 1. Sec-butyllithium (Sec-BuLi) was used to initiate the polymerization of the styrene monomers in tetrahydrofurane (THF) at $-78^{\circ} \mathrm{C}$. After complete conversion of the styrene monomers (about $30 \mathrm{~min}$ ), the reactivity of the active species was decreased using 1,1diphenylethylene followed by the subsequent polymerization of the $2 \mathrm{VP}$ or $4 \mathrm{VP}$ monomers.

The different BCPs were characterized by $1 \mathrm{H}$ NMR $(\delta$ (ppm), $400 \mathrm{MHz}, \mathrm{THF}$ ), and size exclusion chromatography (SEC) in THF. The elution times were converted to molecular weights using a calibration curve based on

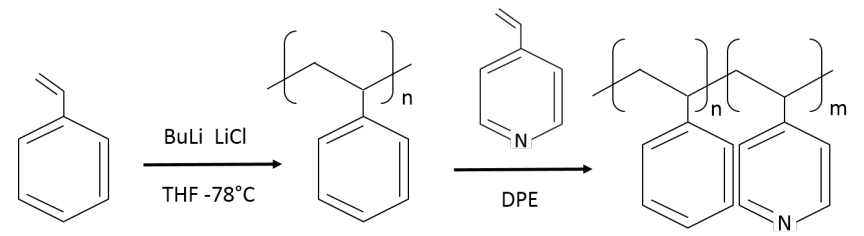

FIG. 1: Scheme of the reaction. Polystyrene is obtained in $\mathrm{THF}$ at $-78^{\circ} \mathrm{C}$ in the presence of $\mathrm{LiCl}$ as additive.

Polystyrene living chains were end-capped with a unit of diphenyl ethylene (DPE) before adding 4 -vinylpyridine (4VP).

low dispersity polystyrene standards. Table I lists the macromolecular parameters of the various BCPs: molar masses $\mathrm{Mn}$ and $\mathrm{Mw}$ were extracted from the SEC analysis while the styrene $(\mathrm{S})$ and pyridine $(\mathrm{P})$ molar fractions were extracted from the NMR analysis.

\section{B. Block copolymer self-assembly and selective hybridization}

Highly regularly organized P4VP dots with a centerto-center distance of $30 \mathrm{~nm}$, are obtained immediately after spin-coating (30 s, $2000 \mathrm{rpm})$ a $0.5 \%$ wt. solution of PS14.7K-b-P4VP6.3k in propylene glycol methyl ether acetate (PGMEA) onto bare silicon wafers (Fig. 2A). In the case of the two higher molecular weight $\mathrm{BCP}$, the self-assembled structures obtained after spin-coating a 2.5 wt.\% BCP solution in toluene (30 s, $4000 \mathrm{rpm})$ are ill-defined due to a low chain mobility, thus inhibiting a fast microphase separation process [28-30]. Therefore, a subsequent solvent vapour annealing (SVA) process was necessary to improve the $\mathrm{BCP}$ ordering. It consists in exposing the as-prepared BCP thin film to vapors of one (or more) solvent. The main effect of the SVA is to swell the BCP film and to give mobility to the polymer chains in order to form equilibrium well-organized structures [31]. After the SVA process, well-organized out of plane cylinders of P2VP and P4VP in a PS matrix are obtained (Fig. 2B and Fig. 2C), with center-to-center distances of $80 \mathrm{~nm}$ (PS150K-b-P2VP32k) and $110 \mathrm{~nm}$ (PS267k-bP4VP177k), respectively. The Fast Fourier Transforms (FFT) of the AFM topographic images, shown as insets in Figs. 2(A)-(C), demonstrate the quality of the final order in the polymer films.

In order to produce the desired metallic arrays, the second step of the fabrication process consists of the selective impregnation of the BCP structure. In this case, the selective incorporation of the gold into the $\mathrm{P} 2 \mathrm{VP}$ domains is insured by the Brønsted base character of the $4 \mathrm{VP}$ and $2 \mathrm{VP}$ units forming pyridium salts in the presence of the tetrachloroauric acid $\left(\mathrm{HAuCl}_{4}\right)$ through the protonation of the pyridine moieties [32]. The polymer films were immersed in the metallic salt precursor solution $\left(\mathrm{HAuCl}_{4}, 1\right.$ wt.\% in milliQ $\left.\mathrm{H}_{2} \mathrm{O}\right)$ for a fixed dura- 
TABLE I: Macromolecular characteristics of the synthetized BCPs. Note that Fraction S is also denoted molar ratio.

\begin{tabular}{l|ccccc} 
Sample & $\mathrm{Mn}(\mathrm{kg} / \mathrm{mol})$ & $\mathrm{Mw}$ & $\mathrm{Mw} / \mathrm{Mn}$ & Fraction S Fraction P \\
\hline (A) PS14.7K-b-P4VP6.3k & 21.0 & 25.9 & 1.23 & 69.9 & 30.1 \\
(B) PS150K-b-P2VP32k & 182.7 & 248.7 & 1.36 & 81.9 & 18.1 \\
(C) PS267k-b-P4VP177k & 443.9 & 500.3 & 1.13 & 58.3 & 41.7
\end{tabular}
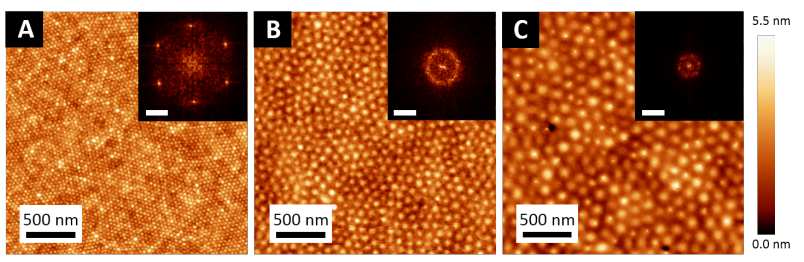

FIG. 2: AFM topographical image of the films of (A) PS14.7K-b-P4VP6.3k out-of-plane cylinders obtained after casting from a 0.5 wt.\% in PGMEA solution (B) PS150K-b-P2VP32k out of plane cylinders obtained after casting from a 2.5 wt.\% in toluene solution and after 16h THF SVA and (C) PS267k-b-P4VP177k out of plane cylinders obtained after casting from a 2.5 wt. $\%$ in toluene solution and after $24 \mathrm{~h}$ THF SVA. The insets show the corresponding FFTs, where the scalebar length is $20 \mu \mathrm{m}^{-1}$.

tion (30 $\mathrm{min})$ to facilitate the ionic interaction between the pyridine and the $\mathrm{Au}(\mathrm{III})$ ions. The penetration of the gold salts is facilitated by the swelling in water of the P4VP and P2VP domains and appears to be homogeneous along the whole film thickness. A final step of $\mathrm{O}_{2}$ RIE (60 W, $60 \mathrm{~s}, 10 \mathrm{sccm}$ ) was performed in order to remove the $\mathrm{BCP}$ template and reduce the metallic salts.

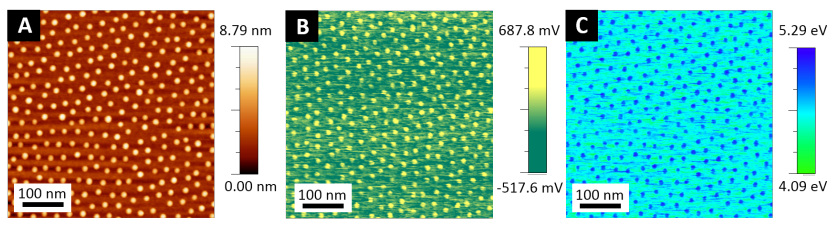

FIG. 3: Kelvin Probe Force Microscopy images of the $\mathrm{Au}$ NPs hexagonal array after $\mathrm{O}_{2}$ RIE treatment of the selectively impregnated PS14.7K-b-P4VP6.3k film. (A)

Topography image; (B) contact potential difference

(CPD) image (in $\mathrm{mV}$ ) between the sample and the microscope tip; (C) work function map (in $\mathrm{eV}$ ) of the sample surface retrieved from the composite topographical and CPD images.

At the end of the process, the decorated surface consists of a hexagonal array of metallic gold NPs deposited on the silicon wafer, which is proven through the Kelvin Probe Force Microscopy [16] images in Fig. 3. The AFM topographic images of the three samples studied further by spectroscopic ellipsometry are shown in Fig. 4 and are found to faithfully reproduce the initial BCP pattern: the gold NPs arrays obtained with the PS14.7K-b-P4VP6.3k film is labelled Sample A, with the PS150K-b-P2VP32k film labelled Sample B and with the PS267k-b-P4VP177k film labelled Sample C. The high degree of organization of the NPs is demonstrated through the corresponding autocorrelation images in Figs. 4(D-F). The Au NP dimensions and the lattice constants are thus established through AFM, and independently through Grazing Incidence Small-Angle X-ray Scattering (GISAXS) and scanning electron microscopy (SEM) (not shown here). Due to tip morphology effects, the AFM can only provide an upper bound value of the in-plane dimension of the gold dots (e.g. maximum radius of $\sim 27 \mathrm{~nm}$ for the Sample C) and a lower bound value of their height (e.g. minimum height of $\sim 25 \mathrm{~nm}$ for Sample C). The center-to-center distances between dots (mostly unaffected by tip effects) are found to be $31 \mathrm{~nm}, 80 \mathrm{~nm}$ and $110 \mathrm{~nm}$, for the gold NPs arrays of Sample A, Sample B and Sample C, respectively.

\section{Spectroscopic ellipsometry}

The optical study of the gold nanostructures deposited on silicon-wafers was performed using variable angle spectroscopic ellipsometry (VASE) in reflection with a phase modulated spectroscopic ellipsometer (UVISEL, from Horiba Scientific) in the spectral range $0.7-4.8$ $\mathrm{eV}$. We used the UVISEL configurations II $\left(\mathrm{A}=45^{\circ} ; \mathrm{M}\right.$ $\left.=0^{\circ}\right)$ and III $\left(\mathrm{A}=45^{\circ} ; \mathrm{M}=45^{\circ}\right)$, where $\mathrm{A}$ and $\mathrm{M}$ denote the azimuthal orientations of the input polarizer and the photoelastic modulator, respectively, with respect to the plane of incidence. Three values of the incidence angle $\theta_{0}=50^{\circ}, 60^{\circ}$ and $70^{\circ}$ were recorded and analysed simultaneously. The spot size was $1 \mathrm{~mm}$ and the measured data were checked to be similar at three different locations on the samples. We acquired the ellipsometric quantities $I_{S}=\sin (2 \psi) \sin (\Delta), I_{C}=\sin (2 \psi) \cos (\Delta)$ and $I_{N}=\cos (2 \psi)$, where $\psi$ and $\Delta$ are the two ellipsometric angles, defined by the ellipsometric ratio $\rho=\frac{r_{p}}{r_{s}}=$ $\tan (\psi) \exp (i \Delta)$, with $r_{p}$ and $r_{s}$ being the complex reflection coefficients of the p-polarized (in the plane of incidence) and the s-polarized (perpendicular to the plane of incidence) electric field components, respectively. Spectroscopic ellipsometry data measured on the bare silicon substrate were initially analysed using the DeltaPsi2 software from Horiba Scientific and the c-Si and $\mathrm{SiO}_{2}$ tabulated dielectric functions and yielded a thickness value of $2.0 \mathrm{~nm}$ for the native silica layer on the surface. 

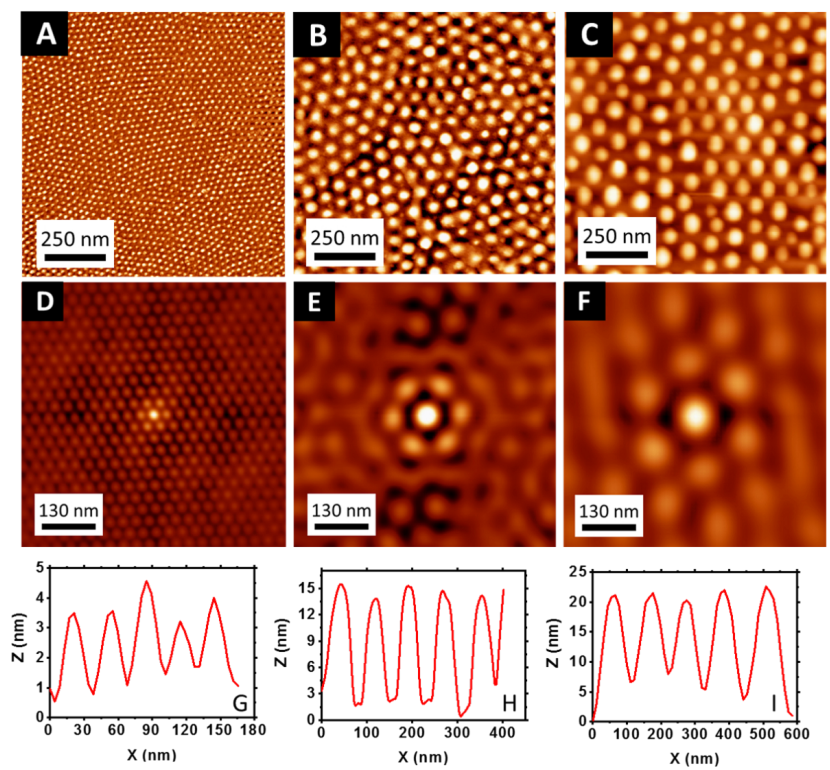

FIG. 4: AFM topographical images (top) and 2D autocorrelation function images (middle) of the Au NPs hexagonal arrays obtained after the $\mathrm{O}_{2} \mathrm{RIE}$ treatment of the selectively impregnated polymer films $(\mathrm{A}, \mathrm{D})$ PS14.7K-b-P4VP6.3k (Sample A) (B,E) PS150K-b-P2VP32k (Sample B) and (C,F) PS267k-b-P4VP177k (Sample C). The corresponding AFM topographical profiles (bottom) of the Au NPs decorated surfaces, though affected by the tip morphology, give an estimate of the dot height: $5 \mathrm{~nm}$, $16 \mathrm{~nm}$ and $25 \mathrm{~nm}$, respectively.

\section{OPTICAL MODELLING THEORY}

\section{A. Standard Bedeaux-Vlieger Model}

The Bedeaux-Vlieger (BV) model [24] uses two surface susceptibilities that are parallel and perpendicular to the surface of the substrate, and will be denoted by $\gamma(\omega)$ and $\beta(\omega)$, respectively. These are related to the particle polarizabilities, and modifies the Fresnel amplitudes of a flat surface to account for the presence of the NP film (or surface roughness), see Eqs. (1) and (2) [23$25,33-35]$. The polarizability of a particle is calculated within the quasi-static approximation by means of using a multipole expansion of the scalar electric potential $[24,33,34,36]$. This is achieved by first calculating, to a high multipole order, the interaction between a single particle and the substrate by the method of images [36]. The single-particle polarizability is next corrected for particle-particle interactions, assuming that this can be done adequately by only including dipolar or quadrupolar interactions. The latter particle polarizabilities calculated within the BV model are used to calculate the surface susceptibilities, and hence the optical response can be calculated $[24,33,34]$. In this work, we have used the BV formalism implemented in the (open source) software GranFilm developed by Simonsen and Lazzari [25]. The advantage of the BV formalism is that it gives a fast calculation (fraction of seconds) of the full spectrum, and thereby allows for fitting morphological parameters. The multipole order of 16 was used in all simulations, while 8 was used in the initial optimization in order to further speed up the calculations.

The reflection amplitude from ambient (medium 0 with refractive index $n_{0}$ (dielectric function $\varepsilon_{0}$, not to be confused with the permittivity of vacuum), an island film (which we will denoted by medium 1, but represented in the BV formalism by the surface susceptibilities) supported by the flat surface of a substrate (medium 2, with refractive index $n_{2}$ (dielectric function $\varepsilon_{2}$ ), is in the $\mathrm{BV}$ formalism given as $[24,25]$

$$
r_{012 s}(\omega)=\frac{n_{0} \cos \theta_{0}-n_{2} \cos \theta_{2}+i \frac{\omega}{c} \gamma(\omega)}{n_{0} \cos \theta_{0}+n_{2} \cos \theta_{2}-i \frac{\omega}{c} \gamma(\omega)} .
$$

Here, $\theta_{0}$ and $\theta_{2}$ are the polar angles of incidence and refraction, respectively, and they are measured positive from the normal to the mean surface[37]. Similarly, the reflection amplitude of an island film for p-polarized incident light, is expressed in the BV model as [24, 25]

$r_{012 p}(\omega)=\frac{\kappa_{-}(\omega)-i \frac{\omega}{c}\left[\gamma(\omega) \cos \theta_{0} \cos \theta_{2}-n_{0} n_{2} \varepsilon_{0} \beta(\omega) \sin ^{2} \theta_{0}\right]}{\kappa_{+}(\omega)-i \frac{\omega}{c}\left[\gamma(\omega) \cos \theta_{0} \cos \theta_{2}+n_{0} n_{2} \varepsilon_{0} \beta(\omega) \sin ^{2} \theta_{0}\right]}$

where

$\kappa_{ \pm}(\omega)=\left(n_{2} \cos \theta_{0} \pm n_{0} \cos \theta_{2}\right)\left(1-\frac{1}{4} \frac{\omega^{2}}{c^{2}} \gamma(\omega) \beta(\omega) \varepsilon_{0} \sin ^{2} \theta_{0}\right)$.

Here, the terms of second or higher order in the surface susceptibilities of Eq. 2b have been neglected[37].

The BV approach uses thus the wavelength, the angle of incidence, the refractive indices of the media involved, in addition to the morphological parameters, in order to calculate the surface susceptibilities, $\gamma(\omega)$ and $\beta(\omega)$. The morphological parameters of the spheroidal island film are the radii of the spheroidal particles that are parallel $\left(R_{x y}\right)$ and perpendicular $\left(R_{z}\right)$ to the surface of the substrate, and the lattice constant, $a$. In addition, how the particle wet the supporting substrate is included by the truncation ratio $\left(0 \leq t_{\text {rat }} \leq 1\right)$ such that the height of the particle is $h=\left(1+t_{\text {rat }}\right) \cdot R_{z}$, see schematic in Fig. 5. These morphological parameters are those that we aim to extract during the inversion of the experimental data sets using the BV model. The BV model only considers a particle directly supported by a substrate, and we need an approach in order to include in the model the additional $\mathrm{SiO}_{2}$ layer underneath each particle (as in Fig. 5(c)). Moreover, due to the particle dimensions, finite size and retardation effects in each particle must be taken into account (as is done in the BV model). 

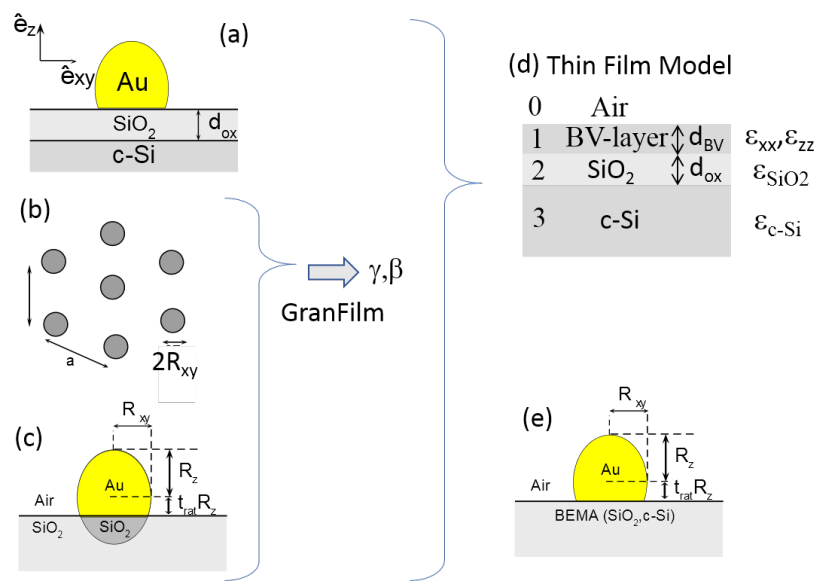

FIG. 5: Schematic diagram of the models. The nanoparticles are presumed formed on top of the native oxide of a c-Si substrate (a), with particle height $R_{z}$ and oxide thickness $d_{o x}$. The particles are presumed in a hexagonal lattice (b) with lattice constant $a$ and particle radius $R_{x y}$. The $\mathrm{BV}$ formalism calculates the surface susceptibilities for particles directly on the substrate with morphological parameters indicated (c). These susceptibilities (assuming only oxide substrate) are then used to calculate the effective dielectric functions for the BV-layer in the thin film multilayer stack (d), with thicknesses $d_{B V}$ and $d_{o x}$. The Bruggeman Effective Medium Approximation (BEMA) are used in the final model in order to calculate the susceptibilities.

\section{B. Thin film Bedeaux-Vlieger Model}

In the approximation of replacing the islands on the substrate by an effective continuous film, the effective dielectric functions $\varepsilon_{x x}=\varepsilon_{y y}$ will depend on $\gamma$ but not $\beta$, in the same way $\varepsilon_{z z}$ will depend on $\beta$ but not $\gamma$. In this approximation the dielectric functions are[25]

$$
\begin{aligned}
\varepsilon_{x x} & =\varepsilon_{0}+\frac{\gamma}{d} \\
\frac{1}{\varepsilon_{z z}} & =\frac{1}{\varepsilon_{0}}-\frac{\beta}{d},
\end{aligned}
$$

where the unknown thickness of the continuous thin film $(d)$ must be chosen independently. We denote $\varepsilon_{0}$ the dielectric function of the ambient incident medium (not to be confused with the permittivity of vacuum), and below we will use $\varepsilon_{2}$ for the dielectric function of the material supporting the particle. The polarizabilities are related to the surface susceptibilities $[24,25]$ and are given as

$$
\begin{aligned}
& \gamma=\rho \alpha_{x x} \\
& \beta=\rho \frac{\alpha_{z}}{\varepsilon_{0}^{2}} .
\end{aligned}
$$

where the number $\rho$ denotes the number of particles per unit area (i.e. $\rho=\frac{2}{\sqrt{3} a^{2}}$ for a hexagonal lattice). The invariant, J, defining the optical properties of the ultrathin film $[23,24,38]$, independent of the choice of the parameter $\mathrm{d}$ is

$J=\int_{0}^{d_{B V}}\left(\varepsilon_{0}+\varepsilon_{2}-\frac{\varepsilon_{0} \varepsilon_{2}}{\varepsilon_{z z}}-\varepsilon_{x x}\right) d z=\left(-\varepsilon_{0} \varepsilon_{2} \beta+\gamma\right) \frac{d_{B V}}{d}$,

One good choice for $\mathrm{d}$ in Eq. 3 is $d=R_{z}$, and is investigated in this work. The multilayer thickness can then be fixed as $d_{B V}=R_{z}$. We have in this paper opted for leaving $d_{B V}$ as a free parameter. It is noted that another natural choice for $\mathrm{d}$ in Eq. 3 is $d=\left(1+t_{r a t}\right) R_{z}=d_{B V}$.

The pragmatic approach used here consists in calculating the dielectric function in Eq. 3 from the surface susceptibilities, themselves calculated from the simplified system of the $\mathrm{Au}$ particle on the $\mathrm{SiO}_{2}$ interface (effectively neglecting image charges in the c-Si bulk), as in Fig. 5c, and finally using $\varepsilon_{0}=1.0$ (air) in Eq. 3. Although this is a debatable simplification, it appears to reproduce reasonably well the lattice constant and lateral particle dimensions, but seems to overestimate the particle height. In order to avoid that the model parameters are compensating for a reduced particle polarizability on the oxide compared to the native oxide/cSi stack, we propose performing the BV calculations using an effective substrate consisting of a Bruggeman Effective Medium[39] layer combining the thin $\mathrm{SiO}_{2}$ oxide layer and the c-Si substrate. The volume fraction $f_{c-S i}=\left(1-f_{\mathrm{SiO}_{2}}\right)$ becomes then another free parameter.

The extracted uniaxial dielectric tensor for the effective BV-layer, see Fig. 5d, then reads

$$
\varepsilon=\left[\begin{array}{ccc}
\varepsilon_{x x} & 0 & 0 \\
0 & \varepsilon_{x x} & 0 \\
0 & 0 & \varepsilon_{z z}
\end{array}\right]
$$

which can be conveniently represented by a standard $4 \times 4$ transfer matrix for a uniaxial layer $\mathbf{T}_{B V}\left(d_{B V}\right)$, with effective thickness $d_{B V}$. The homogeneous underlying layers are given by the isotropic partial transfer matrix $\mathbf{T}_{o x}\left(d_{o x}\right)$ with real thickness $d_{o x}$. The total transfer matrix is then simply given as

$$
\mathbf{T}=\mathbf{L}_{a}^{-1}\left[\mathbf{T}_{B V}\left(d_{B V}\right) \mathbf{T}_{o x}\left(d_{o x}\right)\right]^{-1} \mathbf{L}_{f},
$$

where $\mathbf{L}_{a}$ and $\mathbf{L}_{f}$ are the interface matrices mapping the in plane field components to the s and $\mathrm{p}$ field components at non-normal incidence[40]. The ellipsometric parameters $\left(I_{N}, I_{C}, I_{S}\right)$ or $(\psi, \Delta)$ are then simply calculated from the reflection coefficients

$$
r_{p p}=\frac{T_{11} T_{43}-T_{41} T_{13}}{T_{11} T_{33}-T_{13} T_{31}}, \quad r_{s s}=\frac{T_{21} T_{33}-T_{23} T_{31}}{T_{11} T_{33}-T_{13} T_{31}} .
$$

We have here for convenience and standardization used a Matlab implementation of the Berreman formalism [40, 41], whereas the transfer matrices for the 
isotropic system are the standard $2 \times 2$ scattering transfer matrices[42]. This approach makes it easy to implement the new modelling approach into standard modelling softwares. All simulations and optimizations involving the BV formalism in the current work were performed in a Linux environment with separate calls from Matlab to the GranFilm software, while comparison to standard thin film models used the Complete Ease software.

\section{RESULTS AND DISCUSSION}

We use here the above described model to extract from spectroscopic ellipsometry some morphological information on the 2D hexagonal Au lattices formed as a result of Au impregnation of self-assembled diblock copolymer lattices. The model allows for reproducing the optical response, including phase shifts, at in principle any angle of incidence, and allows through inversion to determine the morphological parameters. The ellipsometric intensities $I_{j}$, where $j=N, C, S$, were modelled in a two step approach. First, the low energy part of the spectrum was used to establish the underlying oxide thickness $d_{o x}$, thus assuming $d_{B V}=0$. This supplied the simulated $I_{j, 0}$. We then also plot for increased visibility the difference spectra: $\Delta I_{j, s i m}=\left(I_{j, s i m}-I_{j, 0}\right)$ and $\Delta I_{j, e x p}=\left(I_{j, e x p}-I_{j, 0}\right)$. Similar differential reflectivity spectra were widely used by Lazzari et al. [43] to obtain high sensitivity, although the clean substrate response was then measured immediately prior to deposition. In a second step, the morphological parameters were found by a simple optimization procedure (fminsearch with bounds available in Matlab) around the plasmon resonance (in the range 1.5-4.5 eV), by minimizing the distance $\left(I_{j, s i m}-I_{j, e x p}\right)^{2}$ with $d_{o x}$ fixed.

\section{A. Modelling response by truncated Au particle on clean c-Si}

A particle deposited directly on a high index substrate results in an optical response strongly modified compared to the case of a low index substrate [25]. The standard $\mathrm{BV}$ formalism implemented in GRANFILM is in principle highly accurate in such a case, as long as i) the particle sizes are much smaller than the wavelength $\lambda$ (quasistatic approximation), ii) the coverage is not too high (i.e. cannot model strong hybridization effects [44]), and the surface lattice resonance connected to the onset of diffraction can be neglected [45], and finally iii) a sufficiently high number of multipoles are included in the calculations [43]. The top figure in Fig. 6 shows both the experimental data $I_{N}, I_{C}, I_{S}$ recorded from Sample $\mathrm{C}$ (big particles) at $70^{\circ}$ angle of incidence, together with the simulated data obtained using parameters roughly optimized, corresponding to a truncated particle directly on c-Si. The parameters were $R_{z}=h=32 \mathrm{~nm}, R_{x y}=22$ $\mathrm{nm}$ and $a_{\text {lat }}=130 \mathrm{~nm}$ (not fitted), resulting in a poor $\chi^{2}=69.8$ in the range $[1.5,4.5] \mathrm{eV}$, see Table II; AuNP on c-Si-0. As expected, the fit is not good compared to e.g. a simple Maxwell Garnett model, but the parameters are still reasonable. The most prominant feature from the simulation is the large dip around $1.5 \mathrm{eV}$ for a completely truncated particle. This feature is strongly enhanced by the presence of the high refractive index substrate, but is strikingly not at all present in the experimental data, although the native oxide is only $2 \mathrm{~nm}$ thick. Such a dip is neither observed in any of the ellipsometric data from $\mathrm{Au}$ nanoparticles we have produced on native oxide c-Si (e.g. similar nanoparticle arrays produced with thin film deposition, Electron Beam Lithography and lift off). Removing the forced truncation of the particle modifies and improves slightly the fit, as seen in the bottom figure in Fig. 6. Here $R_{z}=17 \mathrm{~nm}, R_{x y}=26 \mathrm{~nm}, a_{\text {lat }}=130 \mathrm{~nm}$ (fixed) and $t_{\text {rat }}=0.84$ (fitted), resulting in particle height $h=31 \mathrm{~nm}$, see Table II; AuNP on c-Si-1. The additional substrate induced feature is shifted to higher energies and is now located around $1.8 \mathrm{eV}$, but many new features not present in the data, are present around the resonance at $2.3 \mathrm{eV}$.

\section{B. Morphology and optical response extracted using extended BV formalism and truncated particles}

We now use the extended BV modelling approach presented in section III B in order to better reproduce the optical response of the samples, with the main goal to more accurately determine the morphological parameters. The main hypothesis is here that the BV parameters $\gamma$ and $\beta$ can be calculated from solely the particles on the oxide. In the first case, the particles are presumed completely truncated, i.e. hemispheroids (fixed $t_{r a t}=0$ ). Figure 7 shows both the experimental data $I_{N, C, S}$ recorded from sample C (big particles) at $70^{\circ}$ angle of incidence, together with the simulated data using the fitted parameters, corresponding to a truncated particle on native oxide on c-Si. The results of the latter extended BV modelling are summarized in terms of the morphological parameters given in Table II denoted EBV: Au on ox/c-Si-0. Although the fit is reasonable $\left(\chi^{2}=6.5\right)$, the parameters $d_{B V}, R_{z}$ and $a_{\text {lat }}$ deviate considerably from the parameters measured by SEM and AFM.

\section{Morphology and optical response extracted using extended BV formalism and including wetting $\left(t_{\text {rat }}>0\right)$}

The particle wetting is now included through the parameter $t_{r a t}$, and this parameter is now additionally fitted, i.e. $0 \leq t_{r a t} \leq 1$. The fit is summarized in Table II denoted EBV: AU on ox/cSi-1. The particle truncation ratio converges to $t_{r a t}=0.94$, which means that the par- 
TABLE II: The BV model morphology parameter set $\left(R_{x y}, R_{z}, a, t_{r a t}\right)$ used in the numerical simulations performed using GranFilm, and the thicknesses $d_{B V}$ and $d_{o x}$ used in the extended multilayer formulation of the BV formalism. The derived value of the particle height, $h$, is calculated as $h=\left(1+t_{r a t}\right) \cdot R_{z}$ using the parameters from the BV model (and directly estimated from experimental AFM data). All lengths are given in nanometers. Bold face means that parameters were fitted. The GRANFILM software was used for the purpose of reconstructing the morphological parameters of the samples by fitting the experimental data for the standard ellipsometric quantities presented in Figs. 6-8.

\begin{tabular}{l|cccccccc} 
Model-sample C & $R_{x y}$ & $R_{z}$ & $a_{\text {lat }}$ & $t_{\text {rat }}$ & $h$ & $d_{o x}$ & $d_{B V}$ & $\chi^{2}$ \\
\hline BV: Au on c-Si -0 & $\mathbf{2 2 . 1}$ & $\mathbf{3 1 . 8}$ & 130 & 0 & 31.75 & 0 & - & $\mathbf{6 9}$ \\
BV: Au on c-Si -1 & $\mathbf{2 6 . 4}$ & $\mathbf{1 6 . 6}$ & 130 & 0.84 & 30.5 & 0 & - & $\mathbf{4 8}$ \\
EBV: Au on ox/c-Si-0 & $\mathbf{2 1}$ & $\mathbf{3 6}$ & $\mathbf{1 0 6}$ & 0 & 36 & 2 & $\mathbf{3 0}$ & $\mathbf{6 . 5}$ \\
EBV: Au on ox/c-Si-1 & $\mathbf{2 2}$ & $\mathbf{2 2}$ & $\mathbf{1 2 6}$ & $\mathbf{0 . 9 4}$ & 42.7 & 2 & $\mathbf{1 9 . 8}$ & $\mathbf{4 . 4}$ \\
EBV: Au on ox/c-Si-BEMA & $\mathbf{2 2 . 7}$ & $\mathbf{1 8 . 9}$ & $\mathbf{1 2 6 . 9}$ & $\mathbf{0 . 9 8}$ & 37 & 2 & $\mathbf{1 9 . 6}$ & $\mathbf{3 . 4}$ \\
Structural study & $\leqslant 27$ & - & 110 & - & $\geqslant 25$ & $\mathbf{2}$ & - & -
\end{tabular}

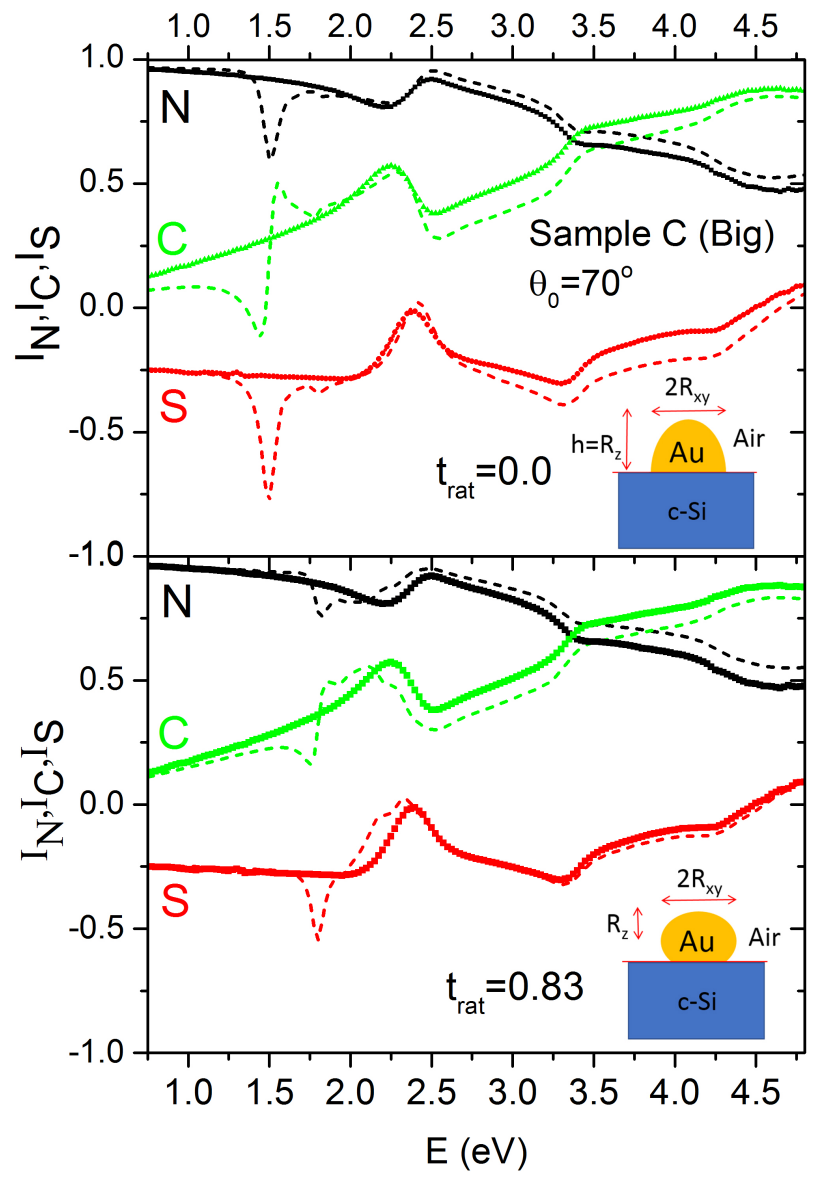

FIG. 6: The experimental intensities $I_{N}$ (black symbols), $I_{C}$ (red symbols) and $I_{S}$ (green symbols) for

Sample C-Big Au particles, as function of photon energy for $\theta_{0}=70^{\circ}$. The dashed lines are the simulated data for a particle directly on the c-Si substrate, using the parameters in Table II for a fully truncated particle,

$t_{\text {rat }}=0$, (top figure), and for a particle with lower wetting, $t_{\text {rat }}=0.84$, (bottom figure). The inset is a graphical visualisation of the particle. The Localized Surface Plasmon Resonance (LSPR) is at $2.3 \mathrm{eV}$ in the experimental data. ticle is hardly wetting the substrate. The $\chi^{2}=4.4$ is improved from 6.5 , but although $R_{z}=22 \mathrm{~nm}$, the total particle height $(\mathrm{h})$, is larger. One hypothesis is that this simplified model compensates for the increased reflectivity due to the presence of the c-Si substrate not accounted for in our pragmatic thin film BV model.

\section{Morphology and optical response extracted using extended BV formalism $\left(t_{\text {rat }}>0\right)$ and BEMA layer}

In order to intuitively introduce the image dipoles also from the underlying c-Si substrate into the model, we propose to replace, in the calculations of the BV parameters $\gamma$ and $\beta$, the pure oxide substrate or the pure $\mathrm{c}-\mathrm{Si}$ substrate by an effective medium of the two. A substrate consisting of a Bruggeman effective medium [39] with $75 \% \mathrm{SiO}_{2}$ and $25 \% \mathrm{c}-\mathrm{Si}$ was found to give the lowest $\chi^{2}$. Figure 8 shows both the experimental data $I_{N, C, S}$ recorded from sample $\mathrm{C}$ (big particles) at $70^{\circ}$ angle of incidence, together with the simulated data using the fitted parameters. The BEMA is found to result in only a minor modification to the BV parameters and a minor improvement of the $\chi^{2}$ (from 4.4 without BEMA to 3.4 with BEMA), as seen from Table II: EBV: $\mathrm{AU}$ on ox/cSi1 and EBV: AU on ox/cSi-BEMA. The simulated curves corresponding to both these models are hardly possible to visually differentiate.

It is noted that the fit result in $R_{z}=18.9 \mathrm{~nm}$, but with total height $\mathrm{h}=37 \mathrm{~nm}$. The height $\mathrm{h}$ is still a factor 2 larger than what was observed with AFM, and either the tip convolution effect underestimated the height in AFM, or the film height extracted from the optical model, to be compared to the AFM height, should rather be related to the continuous thin film height $d_{B V}$. It is noted that for the three EBV models we find that $d_{B V} \approx R_{z}$, which is as expected from the invariant in Eq. 5, and one may in principle simplify the model by setting $d_{B V}=d=R_{z}$.

The model parameters are resulting from the best mathematical solution to the problem, given the model and the described approximations. However, it is encour- 


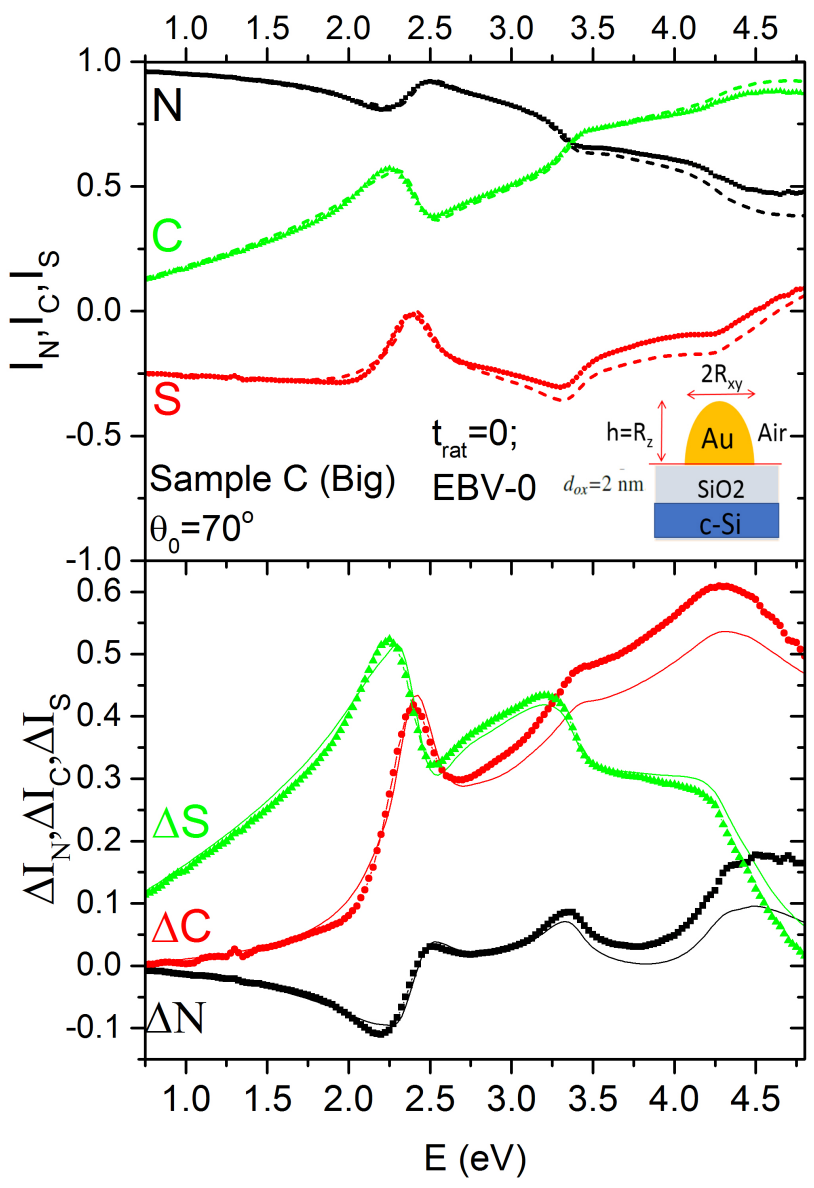

FIG. 7: The top figure shows the experimental intensities $I_{N}$ (black symbols), $I_{C}$ (red symbols) and $I_{S}$ (green symbols) for Sample C-Big Au particles, recorded at $\theta_{0}=70^{\circ}$. The dashed lines are the simulated data using the extended BV model with fixed truncation $\left(t_{\text {rat }}=0\right)$, using the parameters in Table II denoted EBV: AU on ox/cSi-0. The inset is a graphical visualisation of the model.

aging that the morphological parameters are found to be in quite good correspondence to the parameters extracted from SEM and AFM data. The models in sections IV C and IV D were quite similar, but we have here chosen the EBV-BEMA optical model as the most accurate representation of the data.

\section{E. Optical response of the three self-assembled systems}

The experimental and simulated ellipsometric intensities $\Delta I_{j}$, where $j=N, C, S$, for the remaining samples (A)-Small particles and (B)-Medium particles (see Table I), are shown in Figs. 9, while the fit for the (C)-Big particles was shown in Fig. 8. The fitted parameters using the Extended BV formalism with truncation ratio as free parameter, and a BEMA layer in the calculation, are

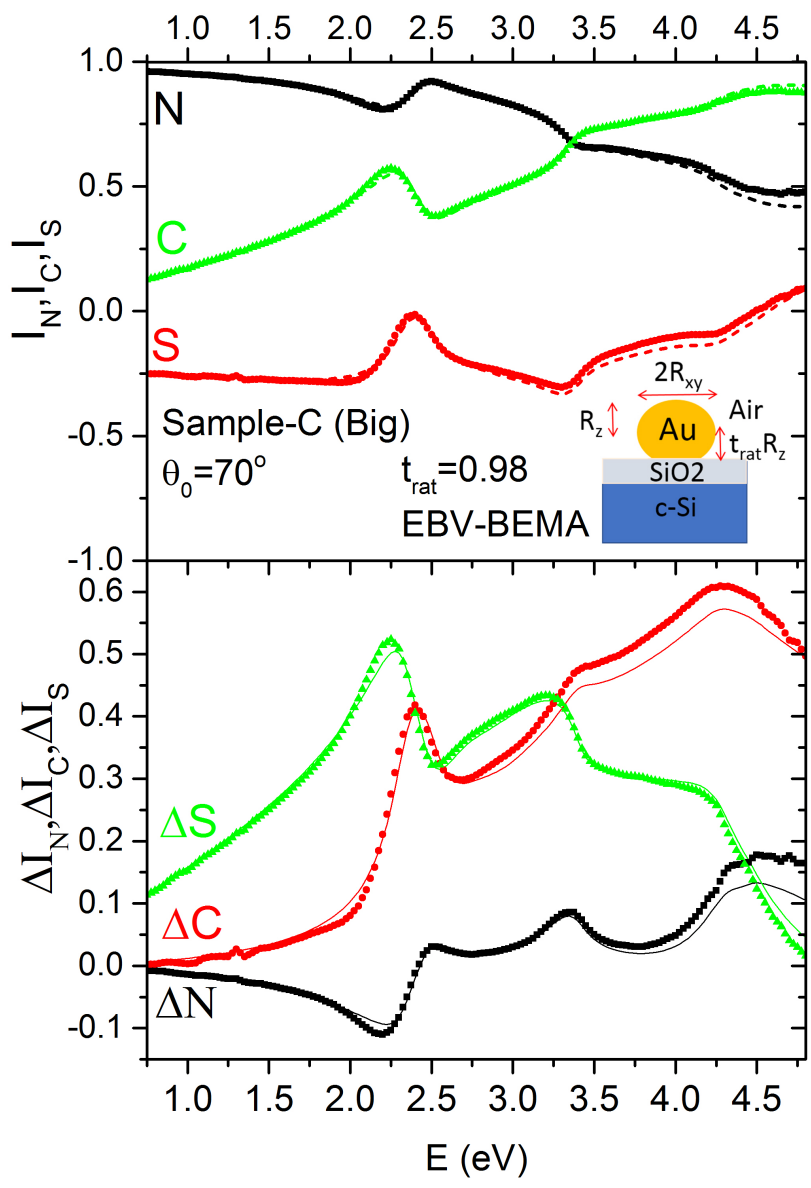

FIG. 8: The top figure shows the experimental intensities $I_{N}$ (black symbols), $I_{C}$ (red symbols) and $I_{S}$ (green symbols) for Sample C-Big Au particles, recorded at $\theta_{0}=70^{\circ}$. The dashed lines are the simulated data using the extended BV model truncation ratio fitted $\left(t_{\text {rat }}=0.98\right)$, using the parameters in Table II denoted EBV: AU on ox/cSi-BEMA. The bottom figure shows the corresponding intensity differences, and the inset is a graphical visualisation of the model.

listed in Table III. It is found that the model results in a solution where all samples are found to weakly wet the $\mathrm{SiO}_{2}$ film.

The calculated surface susceptibilities, presented here as $\hat{\gamma}=\gamma / R_{z}$ and $\hat{\beta}=\beta / R_{z}$, corresponding to these fits are shown in Fig. 10. It is observed that the resonance of $\gamma$ (i.e. the in plane susceptibilities) is always larger in magnitude and slightly red-shifted compared to that of $\beta$. The corresponding particle polarizabilities per volume, here scaled as in the GRANFILM software by $\left(\hat{\alpha}_{x x}=\alpha_{x x} / R_{z}^{3}\right)$ and $\left(\hat{\alpha}_{z z}=\alpha_{z z} / R_{z}^{3}\right)$ are shown in Fig. 11. It is noted that the relationship between $\alpha_{x x}$ and $\gamma$, and $\alpha_{z z}$ and $\beta$ is completely described by Eq.4.

The corresponding dielectric functions for the three samples, using $d=R_{z}$ in Eq. 3 are shown in Fig. 12. The result resembles an harmonic oscillator (i.e. Lorentzian lineshape), similar to what is also expected from the 
TABLE III: The morphology parameter sets $\left(R_{x y}, R_{z}, a, t_{r a t}\right)$ (all lengths in nanometers) used in the numerical simulations performed using GRANFILM, and the thicknesses $d_{B V}$ and $d_{o x}$ used in the extended multilayer formulation of the BV formalism. The GRANFILM software was used for the purpose of reconstructing the morphology parameters of the samples by fitting the experimental data for the standard ellipsometric quantities.

\begin{tabular}{l|cccccccc} 
Sample & $R_{x y}(\mathrm{~nm})$ & $R_{z}$ & $a_{\text {lat }}$ & $t_{r a t}$ & $h$ & $d_{o x}$ & $d_{B V}$ & $\chi^{2}$ \\
\hline A (Small) & $\mathbf{7 . 7}$ & $\mathbf{4 . 6}$ & $\mathbf{3 4 . 3}$ & $\mathbf{1 . 0}$ & 9.2 & 2.0 & $\mathbf{2 . 9}$ & $\mathbf{0 . 0 6}$ \\
B (Medium) & $\mathbf{1 4 . 3}$ & $\mathbf{8 . 4}$ & $\mathbf{8 3}$ & $\mathbf{1 . 0}$ & 16.8 & 2.0 & $\mathbf{6 . 8}$ & $\mathbf{0 . 6}$ \\
C (Big) & $\mathbf{2 2 . 7}$ & $\mathbf{1 8 . 9}$ & $\mathbf{1 2 6 . 9}$ & $\mathbf{0 . 9 8}$ & 37 & 2.0 & $\mathbf{1 9 . 6}$ & $\mathbf{3 . 4}$
\end{tabular}

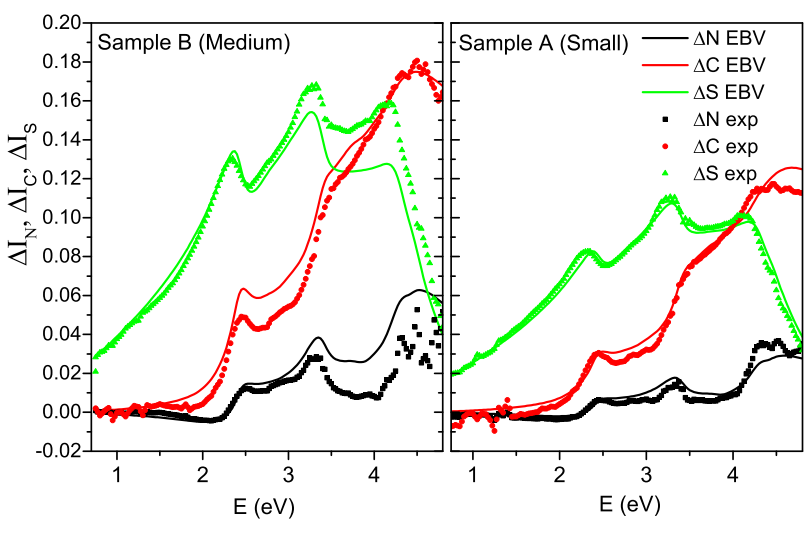

FIG. 9: The figure shows the experimental and simulated intensity differences $\Delta I_{N}$ (black symbols), $\Delta I_{C}$ (red symbols) and $\Delta I_{S}$ (green symbols) for Sample A (Small) (right figure) and sample B (Medium) (left figure), both recorded at $\theta_{0}=70^{\circ}$. The dashed lines are the simulated data using the extended BV model with the parameters in Table III.

isotropic MG-EMA method [46] (black dotted lines in the top figure), see below. There is a small anisotropy in the in- and out-of plane dielectric functions, where in particular the out-of plane one is slightly blue-shifted compared to the in-plane component.

\section{F. Full wave simulations}

Full wave simulations in the frequency domain using COMSOL, was used to simulate the optical response[37, 47 with the optimized morphological parameters. The simulations are obtained by calculating the reflection Jones matrix from two separate TM and TE wave simulations, and finally the optical response in terms of $I_{N, C, S}$. The full lines in Fig. 13 show the simulated data using the parameters in Table II: EBV Au on oxide/c-Si-BEMA. The experimental data are shown as symbols. The location of the Localized Plasmon Resonance appear reasonable, while the amplitude seems too large, but the fit is too poor to be conclusive. Indeed, the relative phase changes were not found accurate (i.e. for $I_{C}$ and $I_{S}$ ), although the relative reflection amplitude ratio $\left(I_{N}\right)$ was in reasonable correspondence to the data (as we observed by reducing the height of the particle). As a comparison,

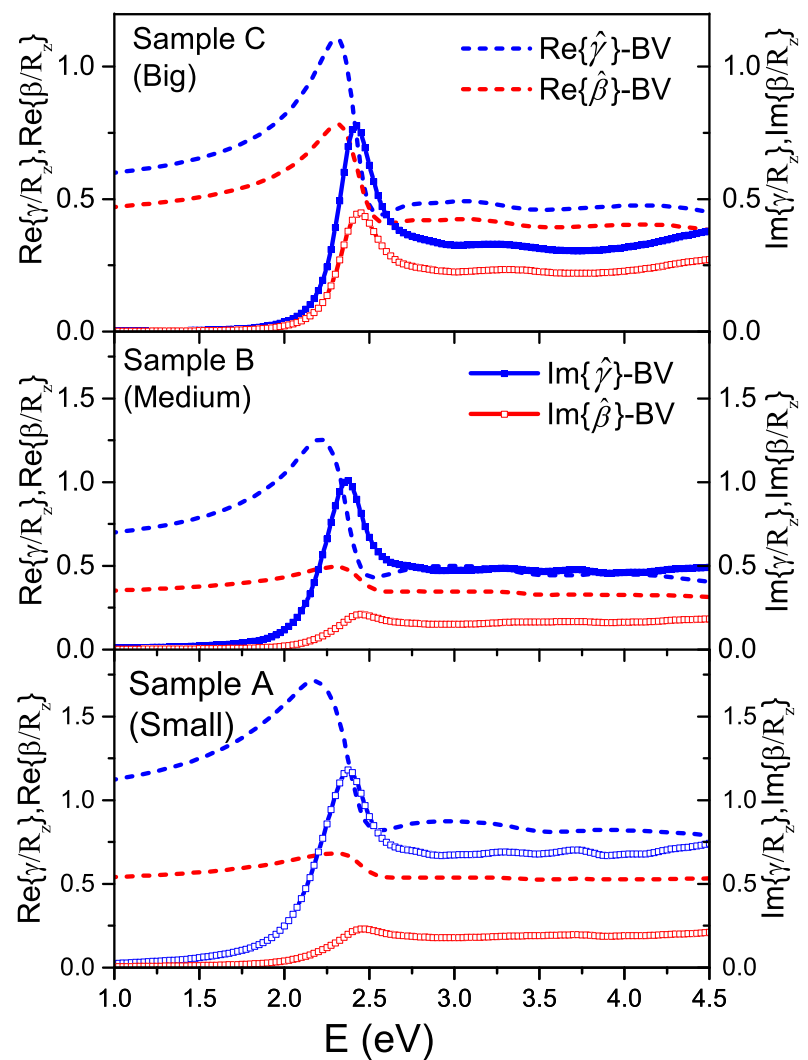

FIG. 10: The surface susceptibilities $\hat{\gamma}=\gamma / R_{z}$ and $\hat{\beta}=\beta / R_{z}$ calculated for sample A (small), sample B (medium) and sample $\mathrm{C}$ (big sized particles), corresponding to the parameters in Table III, and the fits in Figs. 9 and 8.

the simulated data obtained for $t_{r a t}=0$, i.e. using the parameters in Table II: EBV Au on oxide/c-Si-0, are also shown in Fig. 13 (dashed lines). The strong substrate induced response is now observed around $1.9 \mathrm{eV}$, but the fit is overall better at higher energies above the LSPR. It is speculated that static charging mechanisms may be further responsible for complicating the model required for reproducing the measured optical response. One hypothesis is that a Metal Oxide Semiconductor type accumulation layer occurs beneath each particle. Such an issue has been previously described in modelling of infrared spectra of metal films on native oxide-c-Si substrates [48]. Our simulations with optical properties modelled 


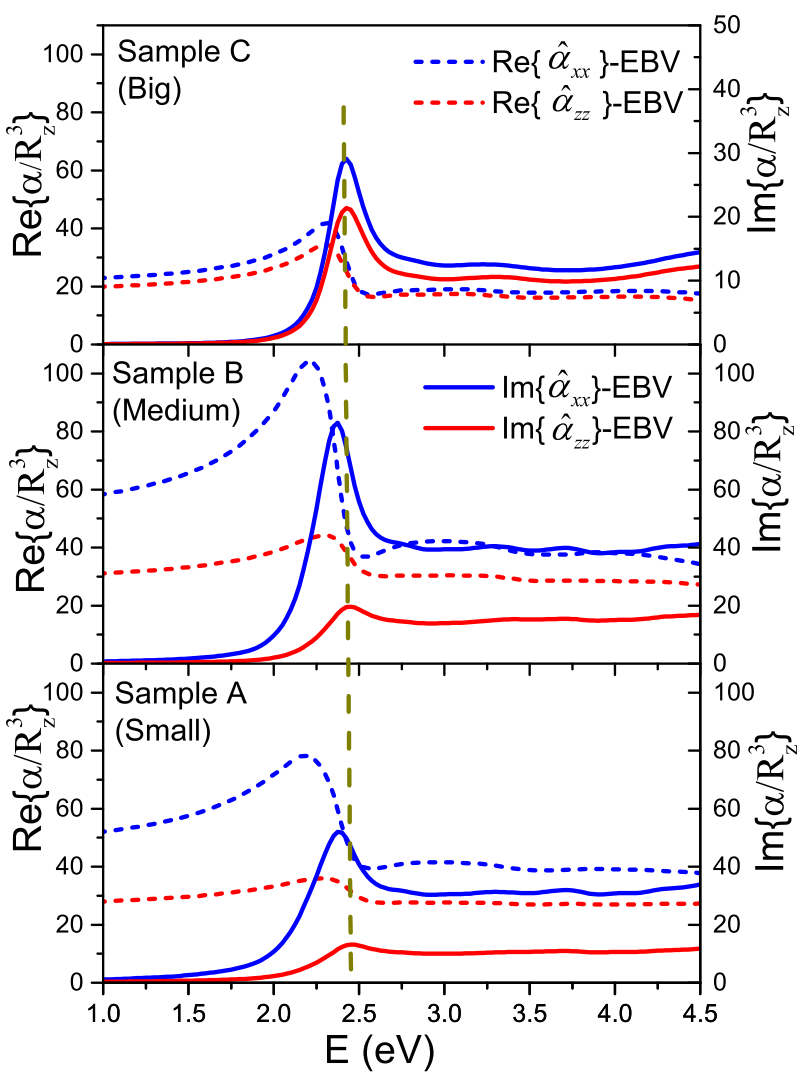

FIG. 11: The in plane $\left(\hat{\alpha}_{x x}=\alpha_{x x} / R_{z}^{3}\right)$ and out of plane $\left(\hat{\alpha}_{z z}=\alpha_{z z} / R_{z}^{3}\right)$ particle polarizabilities per volume, for sample A (small), sample B (medium) and sample C

(big sized particles), corresponding to Fig. 10.

for such an hypothetical accumulation layer was so far inconclusive.

The COMSOL simulations also provide the local electric field distributions, for s- and p-polarized incident light. It was noted that the local fields are mainly concentrated at the oxide particle interface (not shown here). This can be one reason why the pragmatic approach of the extended BV formalism actually does work, i.e. why it is possible to calculate $\beta$ and $\gamma$ for a particle on a $\mathrm{SiO}_{2}$ substrate, although the $\mathrm{SiO}_{2}$ film is only $2 \mathrm{~nm}$ thick.

\section{G. Comparison to the MG-EMA}

The Maxwell Garnett Effective Medium Approximation (MG-EMA) is commonly used to model the dielectric function of free-standing metal particles in a host material. For an anisometric particle of dielectric function $\varepsilon_{a}$, volume fill fraction $f$, in host material $\varepsilon_{b}$ it reads $[23,46,49]$

$$
\frac{\varepsilon_{i}-\varepsilon_{b}}{\varepsilon_{i}+2 \varepsilon_{b}}=\frac{f}{3} \frac{\varepsilon_{a}-\varepsilon_{b}}{\varepsilon_{b}+L_{i}\left(\varepsilon_{a}-\varepsilon_{b}\right)}
$$

It has been shown to supply in practical cases reasonable estimates of the volumetric fill fraction [50]. How-

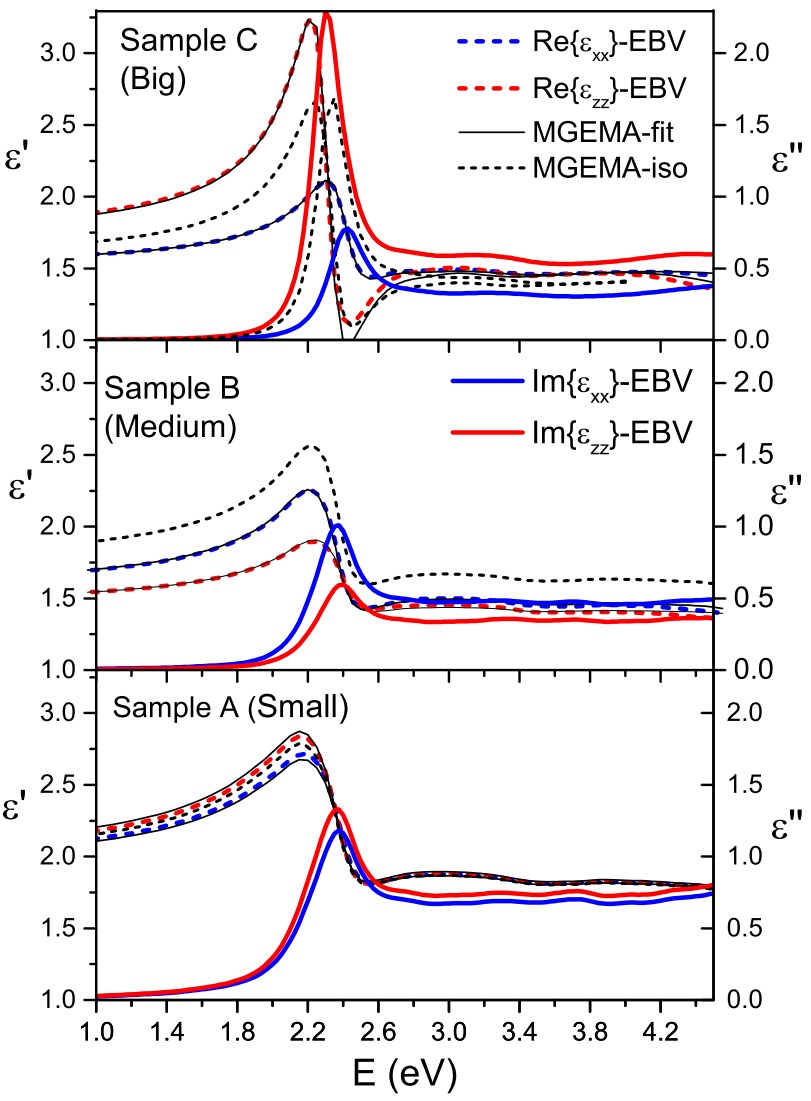

FIG. 12: The dielectric functions $\varepsilon=\varepsilon^{\prime}+i \varepsilon^{\prime \prime}$, for the in plane (blue lines) and out of plane(red lines)

components of the small medium and big size particles, extracted using the extended BV formalism and Eqs. 3 with $d=R_{z}$, corresponding to Figs 10 . The

corresponding parameters are given in Table II. $\varepsilon^{\prime}$ from the isotropic MG-EMA fit (parameters given in

Table V) is plotted in black dashed lines. The thin full lines are the MG-EMA fits to each component with parameters given in Table IV.

ever, when the particle is supported by a surface, the MG-EMA is not strictly valid and it becomes difficult to extract accurate morphological parameters about the nanoparticles [46]. A simple and pragmatic approach assumes an isotropic effective layer, and one may increase the degrees of freedom in the model by both fitting the depolarization factor $L$ and the gold volume fill fraction $f$.

First we investigate how the MG-EMA, with fit parameters $L_{x x, z z}$ and variable volume fill fractions $f_{x x, z z}$, fit the dielectric functions $\varepsilon_{x x}$ and $\varepsilon_{z z}$ previously extracted using the extended thin film BV formalism. The results are summarized in Table IV. We have used the same gold optical properties corrected for finite size effects in both the MG-EMA and the extended BV approach. It is observed that the MG-EMA basically fits well the dielectric functions, with the exception of the $\varepsilon_{z z}$ for sample C (Big). The calculated dielectric functions 


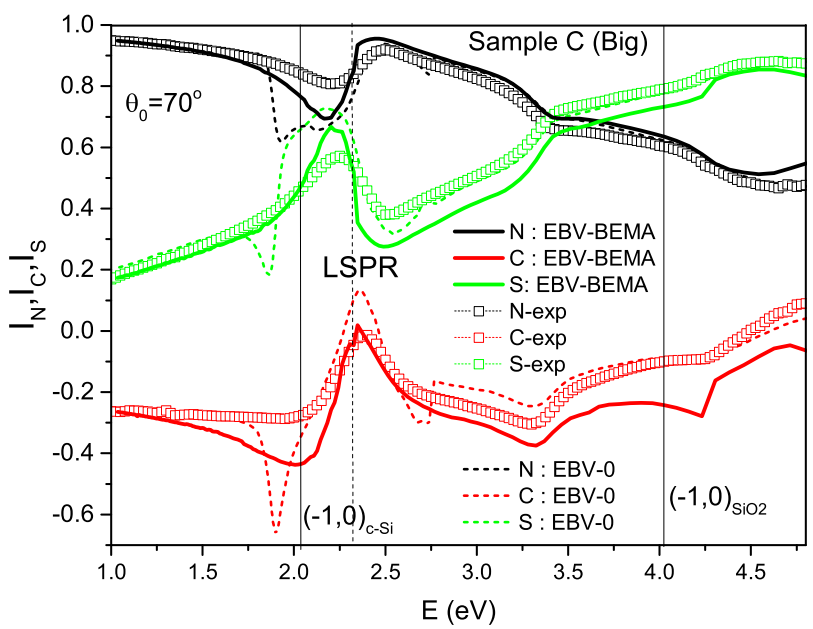

FIG. 13: The experimental intensities $I_{N}$ (black symbols), $I_{C}$ (red symbols) and $I_{S}$ (green symbols) for

Sample C-Big Au particles, as functions of photon energy for $\theta_{i}=70^{\circ}$. The simulated data using COMSOL are shown using the parameters in the model

EBV-BEMA (i.e. $t_{\text {rat }}=0.98$ ) (full lines) and the truncated particles $\left(t_{\text {rat }}=0\right)$ (dotted lines).

using these MG-EMA fits are shown by full thin lines in Fig. 12. The fact that the MG-EMA fits overall so well the data in this case, is speculated to be mainly a result of the poor wetting of the substrate, and hence the optical model for the effective layer can be reproduced by an MG-EMA like response[46]. However, the presence of the substrate clearly causes the depolarization parameters to loose their geometric interpretation, since $2 L_{x}+L_{z}<1$, as also discussed previously by Woormester et al. [46].

We conclude by comparing to a standard isotropic fit to the ellipsometric data, using the MG-EMA model with the parameters $L$ and $f$ for the effective layer. Two sets of fits are performed, one with the thickness of the layer fixed to $d_{B V}$ and the other with the layer thickness being a free parameter. The fit range was limited to $(1.5,4.5)$ $\mathrm{eV}$. The resulting fitted parameters are shown in Table V. The fits are overall good, except at the localized plasmon resonance. Clearly a new effective layer thickness should be fitted, as the response is now assumed isotropic, compared to uniaxial anisotropic for the extended BV formalism. The resulting dielectric functions are similar to the ones extracted by the EBV method, see dotted lines in Fig. 12, the difference being mainly a scaling resulting from the different layer thickness.

The volume fill fraction is given as $f=V_{A U} / V_{\text {hexCell }}$, where $V_{A U}=3 \frac{4 \pi}{3} R_{x y}^{2} R_{z}$ is the volume the spheroidal particles occupies in the hexagonal unit cell for $t_{\text {rat }} \approx 1$, while $V_{\text {hexCell }}=\frac{3 \sqrt{3}}{2} a_{\text {lat }}^{2} h$ is nominally the volume of the unit cell. The volume fill fractions estimated from the EBV parameters are then (12\% for Sample A, $7 \%$ sample B and $9 \%$ for sample C). There is thus up to a factor 2 difference with respect to the results of the simple MG-EMA model,(in models where $t_{r a t} \approx 1$ ). However, within the initial choice $d=R_{z}$ in Eq. 3 we speculate that the height of the hexagonal volume should also be chosen as d, and thus $V_{\text {hexCell }}=\frac{3 \sqrt{3}}{2} a_{\text {lat }}^{2} d$. This results now in a better correspondence with the MG-EMA, i.e. giving (24.3\% for Sample A, $14.3 \%$ sample B and $15.4 \%$ for sample C). It thus appears clear that the choice of $\mathrm{d}$ is going to determine the scaling of the dielectric functions.

TABLE IV: The dielectric functions $\varepsilon_{x x}$ and $\varepsilon_{z z}$ for Sample A-C (Small, Medium and Big) particles have been fitted with a MG-EMA model with free parameters $\mathrm{L}$ and $\mathrm{f}$.

\begin{tabular}{l|cccccc} 
Sample & $L_{x x}$ & $f_{x x}(\%)$ & $L_{z z}$ & $f_{z z}(\%)$ & Mat. & $\chi^{2}\left[10^{-2}\right]$ \\
\hline A & $\mathbf{0 . 3 0 5}$ & $\mathbf{2 4 . 4}$ & $\mathbf{0 . 2 8 6}$ & $\mathbf{2 4 . 1}$ & $A u F z 9$ & $5 / 5$ \\
B & $\mathbf{0 . 2 4 4}$ & $\mathbf{1 3 . 8}$ & $\mathbf{0 . 2 8}$ & $\mathbf{1 2 . 6}$ & $A u F z 14$ & $13 / 15$ \\
C & $\mathbf{0 . 3 0 4}$ & $\mathbf{1 4 . 7}$ & $\mathbf{0 . 1 9 2}$ & $\mathbf{1 3 . 4}$ & $A u$ & $8 / 30$
\end{tabular}

\section{CONCLUSIONS}

We have produced well-organized arrays of $\mathrm{Au}$ nanoparticles by the selective impregnation of the hexagonally-ordered cylindrical poly(vinylpyridine) domains in self-assembled thin films of poly(styrene)-bpoly(vinylpyridine) block copolymers, and we have measured their plasmonic response by variable-angle spectroscopic ellipsometry. A modification to the standard Bedeaux-Vlieger formalism was developed in order to extract the morphological and optical properties of the particulate surface layer on native oxide on $\mathrm{c}-\mathrm{Si}$ from the ellipsometry data. The model allows to extract a uniaxial dielectric function for the effective nanoparticle layer, and can thereby easily be integrated into standard thin film analysis softwares. The result of the new model was compared to Full Wave simulations using COMSOL, the results using a standard MG-EMA model for the effective layer, in addition to the morphological information extracted from the structural study by scanning probe microscopy.

TABLE V: The MG-EMA model parameters $f$ and $\mathrm{L}$ were used to fit directly the ellipsometric parameters in the range $[1.0,4.0] \mathrm{eV}$, for sample A (small) $\mathrm{B}$ (medium) and $\mathrm{C}$ (big), Au particles on native oxide on c-Si. The fitted parameters are shown in bold, while parameters in italic was fixed. For comparison to the BV model, the $d_{o x}$ and $d_{\text {layer }}$ thickness was fixed to those of the extended BV analysis in Table II.

\begin{tabular}{l|cccccc} 
Sample & $L$ & $f(\%)$ & Mat & $d_{\text {layer }}$ & $d_{\text {ox }}$ & $\chi^{2}$ \\
\hline A, free fit & $\mathbf{0 . 2 9}$ & $\mathbf{2 3 . 8}$ & $A u F z 9$ & $\mathbf{2 . 8 8}$ & 2.0 & 3.7 \\
A & $\mathbf{0 . 3}$ & $\mathbf{2 3 . 3}$ & $A u F z 9$ & 2.9 & 2.0 & 3.7 \\
B, free fit & $\mathbf{0 . 2 8}$ & $\mathbf{1 8 . 9}$ & $A u F z 14$ & $\mathbf{5 . 4}$ & 2.0 & 7.1 \\
B & $\mathbf{0 . 3}$ & $\mathbf{1 4 . 5}$ & $A u F z 14$ & 6.9 & 2.0 & 7.9 \\
C, free fit & $\mathbf{0 . 2}$ & $\mathbf{1 1 . 3}$ & $A u$ & $\mathbf{2 5 . 1 3}$ & 2.0 & 15 \\
C & $\mathbf{0 . 1 7}$ & $\mathbf{1 3 . 8}$ & $A u$ & 19.6 & 2.0 & 19
\end{tabular}




\section{APPENDIX}

\section{A. A 2x2 thin film approach}

Within the same pragmatic approximation with respect to calculating the BV parameters $\gamma$ and $\beta$ by neglecting the interface native oxide layer, it is possible to use the $2 \times 2$ scattering matrix formalism [42]. The latter method is briefly discussed. We consider again the system in Fig. 5d , divided into media 0-Air, 1-BV layer, 2 -oxide layer and 3 substrate (c-Si) bulk. The $2 \times 2$ transfer scattering matrix between layer 0 and layer 2 (one for each polarization), is given as:

$$
\mathbf{F}=\frac{1}{t_{012}}\left[\begin{array}{cc}
1 & -r_{210} \\
r_{012} & t_{012} t_{210}-r_{012} r_{210}
\end{array}\right]
$$

where $r_{012}$ and $t_{012}$ are the standard reflection and transmission amplitude coefficients calculated by the BV model, within the same pragmatic approximation as above. These are readily calculated using e.g. the GraNFILM software. However, $r_{210}$ and $t_{210}$ are the amplitude coefficients calculated from the opposite side of the stack. The total scattering matrix for the system in Fig. $5 \mathrm{~d}$ is now simply given by

$$
\mathbf{S}=\mathbf{F L}_{2} \mathbf{I}_{23}
$$

where $\mathbf{L}_{2}$ is the layer matrix for the oxide, and $\mathbf{I}_{23}$ is the interface matrix between the oxide and the c-Si substrate[42]. The overall reflection coefficients are now simply calculated as

$$
r=\frac{S_{21}}{S_{11}} .
$$

The latter approach appears as powerful as the Berreman $4 \times 4$ matrix method using the uniaxial layer, used in the current paper, but is clearly dependent on the same pragmatic hypothesis. It will, eliminate the choice of $d=R_{z}$. However, it requires further work on the numerical implementation in order to correctly calculate $r_{210}$ and $t_{210}$, and is out of the scope of the current paper.

\section{B. The thin film theory with symmetric matrix}

Bohmer et al. [51], Oates et al. [23], and MendozaGalvan et al.[52] used another, but more severe approx- imation, similarly based on the scattering matrix for an unsupported film. This Thin Film Theory is based on first building a symmetric $2 \times 2$ scattering transfer matrix from air (layer 0), the BV layer (layer 1) and to air again $[23,51-53]$

$$
\mathbf{F}=\frac{1}{t_{010}}\left[\begin{array}{cc}
1 & -r_{010} \\
r_{010} & t_{010}^{2}-r_{010}^{2}
\end{array}\right]
$$

By now also introducing an air transfer layer matrix $\mathbf{L}_{\text {air }}$ (with a thickness that is not well defined), the oxide thin film transfer layer matrix $\mathbf{L}_{2}$, in addition to the standard interface matrices between air and oxide $\mathbf{I}_{12}$ and the oxide and the c-Si substrate $\mathbf{I}_{23}$, the final scattering transfer matrix is given as; $\mathbf{S}=\mathbf{F} \mathbf{L}_{a i r} \mathbf{I}_{02} \mathbf{L}_{2} \mathbf{I}_{23}$, and the reflection coefficients are again calculated by Eq. 11 . The hypothesis is that through calculation of $\gamma$ and $\beta$ using the same pragmatic approximation as in Fig. 5 , it is possible to form new reflection and transmission coefficients by letting $\theta_{0}=\theta_{2}$ and $n_{2}=n_{0}$ in the BV formalism in Eqs. 1 and 2 , and the corresponding ones for the transmission coefficients $[24,25]$. This thin film approach was judged much less accurate than the current models, and was not further investigated here.

\section{ACKNOWLEDGEMENTS}

The authors are grateful to Ingve Simonsen and and Synnøva Indrehus for discussions related to GRANFilm. This work was performed within the framework of the LabEx AMADEus ANR-10-LABEX-0042AMADEUS and the EquipEx ELORPrintTec ANR-10EQPX-28-01 with the help of the French state Initiative d'Excellence IdEx ANR-10-IDEX-003-02. It has benefited from an Aurora Hubert Curien Partnership, funded by Norges Forskningsråd (NFR) and by the French ministères des Affaires étrangères et du Développement international (MAEDI) and de l'Education nationale, de l'Enseignement supérieur et de la Recherche (MENESR). We acknowledge the help of Gilles Pécastaings from LCPO. G. F. acknowledges financial support from the Industrial Chair (Arkema/ANR) within the grant agreement no. AC-2013-365.

\section{REFERENCES}

[1] R. P. Feynman, in Miniaturization, edited by H. D. Gilbert (Reinhold Publ. Corp, New York, 1961) pp. 282296.

[2] G. Whitesides, Nature Biotechnology 21, 1161 (2003).

[3] S. Lal, S. Link, and N. Halas, Nature Photonics 1, 641 (2007).
[4] A. Zayats, I. Smolyaninov, and A. Maradudin, Physics Reports 408, 131 (2005).

[5] W. Wang, M. Ramezani, A. I. Väkeväinen, P. Törmä, J. G. Rivas, and T. W. Odom, Materials today 21, 303 (2018).

[6] M. I. Stockman, Physics Today 64, 39 (2011). 
[7] J. Wang, Frontiers of Optoelectronics 7, 320 (2014).

[8] V. Ponsinet, A. Baron, E. Pouget, Y. Okazaki, R. Oda, and P. Barois, EPL 119, 14004 (2017).

[9] A. Klinkova, R. M. Choueiri, and E. Kumacheva, Chemical Society Reviews 43, 3976 (2014).

[10] M. Grzelczak and L. M. Liz-Marzan, Langmuir 29, 4652-4663 (2013).

[11] F. S. Bates and G. H. Fredrickson, Physics Today 52, 32 (1999).

[12] I. W. Hamley, "The physics of block copolymers," (1998).

[13] F. S. Bates and G. H. Fredrickson, Annual Review of Physical Chemistry 41, 525 (1990).

[14] X. Wang, K. Ehrhardt, C. Tallet, M. Warenghem, A. Baron, A. Aradian, M. Kildemo, and V. Ponsinet, Optics and Laser Technology 88, 85 (2017).

[15] J. Toudert, X. Wang, C. Tallet, P. Barois, A. Aradian, and V. Ponsinet, ACS Photonics 2, 1443 (2015).

[16] A. Alvarez-Fernandez, K. Aissou, G. Pécastaings, G. Hadziioannou, G. Fleury, and V. Ponsinet, Nanoscale Advances 1, 849 (2019).

[17] F. Aubrit, F. Testard, A. Paquirissamy, F. Gobeaux, X. Wang, F. Nallet, P. Fontaine, V. Ponsinet, and P. Guenoun, Journal of Materials Chemistry C 6, 8194 (2018).

[18] S. Salvatore, A. Demetriadou, S. Vignolini, S. S. Oh, S. Wuestner, N. A. Yufa, M. Stefik, U. Wiesner, J. J. Baumberg, O. Hess, et al., Advanced Materials 25, 2713 (2013).

[19] S. Lamarre, A. Sarrazin, J. Proust, H. Yockell-Lelièvre, J. Plain, A. Ritcey, and T. Maurer, Journal of Nanoparticle Research 15, 1 (2013).

[20] J. Y. Kim, H. Kim, B. H. Kim, T. Chang, J. Lim, H. M. Jin, J. H. Mun, Y. J. Choi, K. Chung, J. Shin, S. Fan, and S. O. Kim, Nature Communications 7, 12911 (2016).

[21] J. C. M. Garnett, Philosophical Transactions of the Royal Society of London. Series A, Containing Papers of a Mathematical or Physical Character (1896-1934) 203, 385 (1904).

[22] T. Yamaguchi, S. Yoshida, and A. Kinbara, Thin Solid Films 21, 173 (1974).

[23] T. Oates, H. Wormeester, and H. Arwin, Prog. Surf. Sci. 86, 328 (2011)

[24] D. Bedeaux and J. Vlieger, Optical Properties Of Surfaces, 2nd ed. (Imperial College Press, London, 2004).

[25] R. Lazzari and I. Simonsen, Thin Solid Films 419, 124 (2002)

[26] S. K. Varshney, X. F. Zhong, and A. Eisenberg, Macromolecules 26, 701 (1993), https://doi.org/10.1021/ma00056a022.

[27] Z. Gao, S. K. Varshney, S. Wong, and A. Eisenberg, Macromolecules 27, 7923 (1994), https://doi.org/10.1021/ma00104a058.

[28] M. B. Runge and N. B. Bowden, Journal of the American Chemical Society 129, 10551 (2007), pMID: 17685524, https://doi.org/10.1021/ja072929q.

[29] O. Ikkala and G. ten Brinke, Science 295, 2407 (2002), http://science.sciencemag.org/content/295/5564/2407.full.pdf.
[30] E. Kim, H. Ahn, S. Park, H. Lee, M. Lee, S. Lee, T. Kim, E.-A. Kwak, J. H. Lee, X. Lei, J. Huh, J. Bang, B. Lee, and D. Y. Ryu, ACS Nano 7, 1952 (2013), pMID: 23441640, https://doi.org/10.1021/nn3051264.

[31] C. Sinturel, M. Vayer, M. Morris, and M. A. Hillmyer, Macromolecules 46, 5399 (2013), https://doi.org/10.1021/ma400735a.

[32] M. Antonietti, E. Wenz, L. Bronstein, and M. Seregina, Advanced Materials 7, 1000 (1995), https://onlinelibrary.wiley.com/doi/pdf/10.1002/adma.19950071205.

[33] I. Simonsen, R. Lazzari, J. Jupille, and S. Roux, Phys. Rev. B 61, 7722 (2000).

[34] R. Lazzari, I. Simonsen, D. Bedeaux, J. Vlieger, and J. Jupille, Eur. Phys. J. B 24, 267 (2001).

[35] A. Mendoza-Galván, K. Järrendahl, A. Dmitriev, T. Pakizeh, M. Käll, and H. Arwin, Opt. Express 19, 12093 (2011).

[36] J. Jackson, Classical Electrodynamics, 3rd ed. (John Wiley \& Sons, New York, 2007).

[37] M. Kildemo, J.-P. Banon, A. Baron, B. B. Svendsen, T. Brakstad, and I. Simonsen, Applied Surface Science 421, 593 (2017).

[38] J. Lekner, Pure and Applied Optics: Journal of the European Optical Society Part A 3, 821 (1994).

[39] H. G. Tompkins and E. A. Irene, Handbook of Ellipsometry (William Andrew Inc., New York, 2005).

[40] M. Schubert, Phys. Rev. B 53, 4265 (1996).

[41] D. W. Berreman, J. Opt. Soc. Am. 62, 502 (1972).

[42] R. M. A. Azzam and N. M. Bashara, Ellipsometry and Polarized Light (North-Holland Publishing Company, Amsterdam, 1977).

[43] R. Lazzari, S. Roux, I. Simonsen, J. Jupille, D. Bedeaux, and J. Vlieger, Phys. Rev. B 65, 235424 (2002).

[44] E. Prodan, C. Radloff, N. J. Halas, and P. Nordlander, Science 302, 419 (2003), https://science.sciencemag.org/content/302/5644/419.full.pdf.

[45] V. Kravets, A. Kabashin, W. Barnes, and A. Grigorenko, Chem. Rev. 118, 5912 (2018).

[46] H. Wormeester, E. S. Kooij, and B. Poelsema, physica status solidi (a) 205, $756 \quad$ (2008), https://onlinelibrary.wiley.com/doi/pdf/10.1002/pssa.200777740.

[47] P. M. Walmsness, T. Brakstad, B. B. Svendsen, J.-P. Banon, J. C. Walmsley, and M. Kildemo, J. Opt. Soc. Am. B 36, E78 (2019).

[48] M. Schubert, C. Bundesmann, H. v. Wenckstern, G. Jakopic, A. Haase, N.-K. Persson, F. Zhang, H. Arwin, and O. Inganäs, Applied Physics Letters 84, 1311 (2004), https://doi.org/10.1063/1.1649822.

[49] S. Maier, Plasmonics: Fundamentals and Applications (Springer, New York, 2007).

[50] J. Vieaud, O. Merchiers, M. Rajaoarivelo, M. Warenghem, Y. Borensztein, V. Ponsinet, and A. Aradian, Thin Solid Films 603 (2016), 10.1016/j.tsf.2016.02.022.

[51] M. R. Böhmer, E. A. van der Zeeuw, and G. J. Koper, Journal of Colloid and Interface Science 197, 242 (1998).

[52] A. Mendoza-Galván, K. Järrendahl, A. Dmitriev, T. Pakizeh, M. Käll, and H. Arwin, Opt. Express 20, 29646 (2012).

[53] D. M. Spink, Journal of Modern Optics 34, 1263 (1987). 